
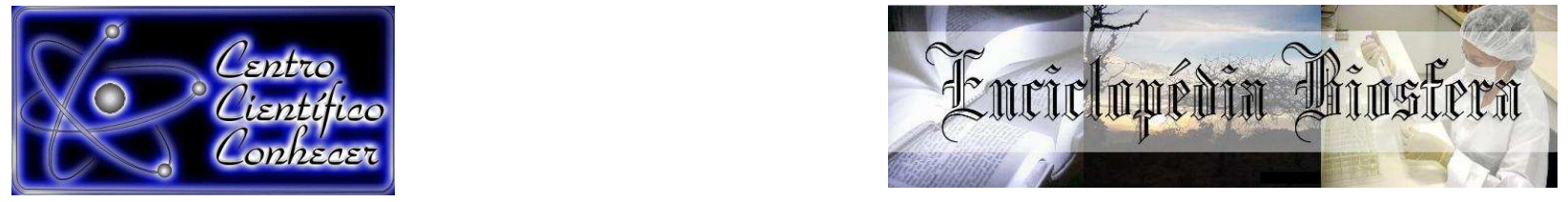

\title{
RENTABILIDADE E CUSTO DE PRODUÇÃO DO CULTIVO DE MELANCIA IRRIGADA NO NORDESTE DO ESTADO DE MATO GROSSO
}

\author{
Givanildo Sousa Gonçalves ${ }^{1}$, Joel Carlos Alves ${ }^{2}$, Aureane Cristina Teixeira Ferreira ${ }^{1}$, \\ Ricardo Adriano Felito ${ }^{1}$, Oscar Mitsuo Yamashita ${ }^{3}$ \\ 1 Mestrando da Universidade do Estado de Mato Grosso, \\ (aurianeferreira@hotmail.com), Alta Floresta MT- Brasil \\ 2 Graduado em Licenciatura em Ciências Agrícolas pelo Instituto Federal de Mato \\ Grosso, Confresa MT- Brasil \\ 3 Professor Doutor da Universidade do Estado de Mato Grosso, Alta Floresta MT- \\ Brasil
}

\section{Recebido em: 08/04/2016 - Aprovado em: 30/05/2016 - Publicado em: 20/06/2016 DOI: 10.18677/Enciclopedia_Biosfera_2016_101}

\begin{abstract}
RESUMO
Dentre os cultivos explorados economicamente no estado de Mato Grosso, a melancia é uma cultura que se destaca a nível de agricultura familiar. Na região nordeste do estado, tradicionalmente se cultiva mandioca e cana-de-açúcar. Mas, muitos agricultores familiares, têm investido na produção de grãos como o milho, arroz e também espécies frutíferas como banana e melancia. O cultivo de melancia se destaca pela capacidade de produção em curto período de tempo e adaptação às condições climáticas desta mesorregião matogrossense. Entretanto, pesquisadores tem verificado nesta região, que a cultura tem apresentado baixa produção, sendo necessário importar o fruto de outros estados para suprir a demanda local, elevando assim o preço do produto para o consumidor. Este trabalho teve como objetivo avaliar a produtividade e viabilidade econômica do cultivo de melancia, cultivada em sistema de irrigação por gotejamento em Confresa-MT. A cultura foi conduzida em uma área de 0,5 hectares em área experimental do IFMT de Confresa-MT. A produtividade obtida foi de $48.000 \mathrm{~kg} \mathrm{ha}^{-1}$, verificou-se uma relação beneficio/custo de 1,86; o ponto de nivelamento foi de $25.737,74 \mathrm{~kg} \mathrm{ha}^{-1}$ e a margem de segurança foi de $-0,46 \%$, sendo superior a algumas regiões polos de produção de melancia. Nas condições que foi realizado a presente pesquisa é possível concluir que o cultivo da melancia na região de Confresa-MT é viável, com grande potencial de geração de renda, retorno financeiro relativamente médio e com bons indicadores econômicos.
\end{abstract}

PALAVRAS-CHAVE: fruticultura, irrigação, viabilidade econômica

\section{PROFITABILITY AND COST OF WATERMELON IRRIGATED CROP PRODUCTION IN NORTHEAST OF MATO GROSSO}

\begin{abstract}
Among the crops economically exploited in the state of Mato Grosso, watermelon is a culture that emphasizes the level of family farming. In the northeastern state, ENCICLOPÉDIA BIOSFERA, Centro Científico Conhecer - Goiânia, v.13 n.23; p.1165 2016
\end{abstract}


traditionally the farmers grow cassava and sugarcane. But many famliares farmers have invested in the production of grains such as corn, rice and also fruit trees like bananas and watermelon. The watermelon cultivation stands out for production capacity in a short period of time and adapt to climate conditions of this middle region of Mato Grosso. However, researchers have found in this region, that crop has shown low production, being necessary to import fruit from other states to supply the local demand, thus raising the price of the product to the consumer. This study aimed to evaluate the productivity and economic viability of watermelon, grown in drip irrigation system Confresa-MT. The crop was grown in an area of 0.5 hectares in the experimental area of IFMT Confresa-MT. The obtained productivity was $48,000 \mathrm{~kg}$ $\mathrm{ha}^{-1}$, there was a benefit/cost ratio of 1.86 ; leveling point was $25,737.74 \mathrm{~kg} \mathrm{ha}^{-1}$ and the safety margin was $-0.46 \%$, exceeding some regions of watermelon production centers. The conditions which was conducted this research we can conclude that the watermelon cultivation in Confresa-MT region is feasible, with great potential for income generation, relatively average financial returns and good economic indicators.

KEYWORDS: Economic viability, fruit growing, irrigation.

\section{INTRODUÇÃO}

A melancia (Citrullus lanatus (Thunb.) Matsum e Nakai) é cultivada em todo o mundo. É uma espécie frutífera que pertence à família das cucurbitáceas, e tem como centro de origem o continente africano. É uma planta com ciclo de vida de 90 dias, sendo que sua colheita pode ser realizada a partir dos 65 dias. Possui hábito de crescimento rasteiro, com diversas ramificações e seu sistema radicular desenvolve-se nos primeiros $30 \mathrm{~cm}$ do solo (BHERING et al., 2003).

De acordo com a FAO (2016), a cultura foi cultivada em 3,12 milhões de hectares em diversos países do mundo, alcançando uma produção de 109,6 milhões de toneladas. Em 2014, o Brasil produziu 2.171.288 toneladas de frutos de melancia em 94.929 hectares. A região Nordeste é a principal produtora com $27 \%$ da produção nacional, sendo que dentre os principais produtores dessa região, destacam-se os estados da Bahia (253.010 ton) e Rio Grande do Norte (121 688 ton). Neste mesmo período, o estado do Mato Grosso produziu 38.653 toneladas de frutos de melancia em uma área de $1.739 \mathrm{ha}^{-1}$, com rendimento médio de $22.227 \mathrm{~kg}$ ha $^{-1}$ (IBGE, 2015).

A melancia está entre os produtos que em 2014 apresentaram o maior valor da produção já experimentado por sua série histórica em reais (IBGE, 2015). Na temporada 2014/15, o Brasil exportou 33,5 mil toneladas da fruta, aumento de 6,2\% frente à safra 2013/14. Em receita, o montante gerado foi de 17,6 milhões de US\$, alta de 6,8\%. (CEPEA, 2015).

Os frutos da melancia são utilizados especialmente para consumo in natura. Pode ser cultivada tanto em condições de sequeiro como também sob irrigação. Seus frutos são considerados de expressiva importância para o agronegócio brasileiro, pois, mesmo sendo cultivada praticamente na sua totalidade em pequenas propriedades, proporcionando rendimentos financeiros interessantes. Além disso, é uma cultura que absorve grande quantidade de mão de obra, devido à necessidade de cuidados e práticas manuais, contribuindo assim para a geração de 
empregos no campo e no seu processamento, para comercialização (ASSUNÇÃO et al., 2014).

A região nordeste do estado de Mato Grosso se destaca pela produção de grãos, caracterizando-se por ser constituída por propriedades rurais que exploram o cultivo de milho, arroz e cana-de-açúcar. Entretanto, muitos pequenos produtores têm desenvolvido atividades relacionadas ao cultivo de hortaliças, plantas extrativas e frutíferas, tais como mandioca e banana (DEEPASK, 2016). Destas, o cultivo de melancia tem-se tornado opção interessante para os produtores. Entretanto, verificase que os produtores que a cultivam, tem obtido baixa produtividade. Essa baixa produção se deve principalmente pelas dificuldades de cultivo e restrições de solo, tais como: elevada acidez e baixa fertilidade, altas infestações de pragas e doenças, reduzido conhecimento técnico do cultivo e também o oneroso custo de produção.

Assim, no mercado local a demanda é suprida pela importação deste fruto de outros estados como Goiás e Tocantins. Essa situação eleva significativamente o preço do produto para o consumidor. Para uma atividade ser considerada lucrativa do ponto de vista econômico, é necessário proporcionar alta rentabilidade econômica e produtiva (SANDRI et al., 2014). Para uma tomada de decisão do que cultivar é necessário o conhecimento dos custos de produção e rentabilidade que permeiam todas as etapas de exploração econômica da cultura (ARAÚJO et al., 2007).

Dessa forma, objetivou-se estimar os custos de produção e a rentabilidade da exploração econômica do cultivo irrigado de melancia, cultivar Crimson select plus, no município de Confresa-MT, no período seco.

\section{MATERIAL E MÉTODOS}

A unidade de análise e estudo foi uma propriedade de agricultura familiar localizada na zona rural (1041'03"latitude Sul e 5194'05"longitude Norte) do município de Confresa - MT. A região possui clima definido como equatorial quente e úmido, com nítida estação seca bem definida em parte do ano. A precipitação anual gira em torno de $2.000 \mathrm{~mm}$ e a temperatura média anual é de $27^{\circ} \mathrm{C}$ (DBCITY, 2016).

$\mathrm{Na}$ área experimental, foram coletadas amostras de solo e realizada análise química e física na profundidade de $0-20 \mathrm{~cm}$, para determinação de possíveis deficiências de macro e micro nutrientes, níveis de saturação por base e capacidade de troca de cátions. Os resultados das análises físicas e químicas foram: $\mathrm{pH}\left(\mathrm{CaCl}_{2}\right)$ : 4,6; Mat. Org.: 1,0\%; $\mathrm{Al}^{+3}: 0,3$ meq $100 \mathrm{~cm}^{3} ; \mathrm{Ca}^{+2}: 0,7 \mathrm{meq} 100 \mathrm{~cm}^{-3} ; \mathrm{Mg}^{+2}: 0,2 \mathrm{meq}$ $100 \mathrm{~cm}^{-3}$; P: $1,00 \mathrm{mg} \mathrm{dm}^{-3}$; K: 32,1 $\mathrm{mg} \mathrm{dm}^{-3}$; areia: $81 \%$; silte: $7 \%$ e argila $12 \%$. A área experimental total atingiu dimensões de $50 \times 100 \mathrm{~m}$ (0,5 hectares).

O preparo da área experimental foi realizado com duas arações e duas gradagens, pois a área era anteriormente explorada com criação de gado. As covas foram preparadas nas dimensões de 0,4 x 0,4 x 0,4 m, com espaçamento de 5,0 m entre linhas e $1,0 \mathrm{~m}$ entre covas na linha de plantio. Estas foram previamente corrigidas com calcário, elevando-se a saturação de base para 70\% (RIBEIRO et al., 1999).

Foi realizada adubação seguindo recomendação proposta por RIBEIRO et al. (1999) para a cultura da melancia, onde o fornecimento de fósforo foi realizado em uma única dose no momento da semeadura com a aplicação de $120 \mathrm{~kg} \mathrm{ha}^{-1}$ de $\mathrm{P}_{2} \mathrm{O}_{5}$ 
(MAP). Foram aplicados $120 \mathrm{~kg} \mathrm{ha}^{-1}$ de potássio (KCl), com aplicação de metade da dose via preparo da cova, e metade parcelada em cinco adubações de cobertura aos 20, 30, 40, 50, 60 dias após a semeadura. O nitrogênio (ureia) foi aplicado em cobertura na proporção de $120 \mathrm{~kg} \mathrm{ha}^{-1}$, dividido em cinco aplicações aos 20, 30, 40, 50, 60 dias após a semeadura.

A aplicação de micronutrientes foi realizada com base na exigência de boro e zinco da cultura e nos teores contidos no solo, sendo utilizado na adubação o formulado FTE BR 12, aplicando-se $60 \mathrm{~kg} \mathrm{ha}^{-1}$. Também foi adicionado ao solo 15 litros de esterco bovino curtido por cova, a fim de melhorar a retenção de água e de disponibilidade de nutrientes.

A cultivar de melancia utilizada foi a Crimson select plus. Distribuíram-se quatro sementes por cova, na profundidade de $3,0 \mathrm{~cm}$. Para controle de doenças e insetos, foram utilizados fungicidas e inseticidas conforme as recomendações técnicas da EMBRAPA (2014) para a cultura e dos produtos. Após 10 dias, foi realizado desbaste mantendo-se apenas as duas plantas mais vigorosas por cova. Também foram realizadas duas capinas manuais, além da realização de condução e orientação dos ramos das plantas.

Para o fornecimento de água, foi utilizada irrigação localizada por gotejamento com vazão de $20 \mathrm{~L} \mathrm{~h}^{-1}$, com turno de rega de 48 horas, sendo a lâmina total de irrigação do cultivo $500 \mathrm{~mm}$. Um motor estacionário modelo Yanmar NS B12 com uma bomba acoplada foi utilizado como fonte de energia para o sistema de irrigação.

A colheita dos frutos foi realizada a partir dos 75 dias após a semeadura, sendo contabilizados todos os frutos com massa igual ou superior a cinco quilos. Para a análise dos custos de produção e da viabilidade econômica da melancia foi utilizado o modelo de custo operacional desenvolvido pelo Instituto de Economia Agrícola de São Paulo (MATSUNAGA et al., 1976), aplicado em estudos de viabilidade de melancia por ARAÚJO (2007) no Submédio São Francisco.

\section{RESULTADOS E DISCUSSÃO}

As condições climáticas são favoráveis para o cultivo da melancia na região de Confresa, permitindo seu desenvolvimento e produção. As plantas cresceram e se ramificaram de maneira adequada, sem quaisquer empecilhos para a reprodutibilidade.

A melancia é uma planta de ciclo de cultivo relativamente curto, se comparado a outras espécies frutíferas de consumo in natura. Essa característica torna-se interessante para a exploração em pequenas escalas, como é o caso de agricultores familiares, pois estes apresentam características como a baixa capacidade de aquisição de recursos a curto prazo (ASSUNÇÃO et al., 2014).

A produtividade média foi de 48 ton $\mathrm{ha}^{-1}$ de frutos (Tabela 2). $\mathrm{O}$ fornecimento de água na quantidade descrita na metodologia foi suficiente para suprir a demanda hídrica da cultura, permitindo que esta atingisse esta capacidade produtiva. A água é fator determinante para a produção vegetal, especialmente para as espécies frutíferas cujo fruto acumula muita água durante o seu desenvolvimento, como é o caso da melancia e outras espécies como melão e abacaxi (CAMPELO et al., 2014)

Essa produção foi superior à relatada por SANDRI et al. (2014), em experimento realizado em Anápolis-GO, em que, ao testarem lâminas e sistemas de irrigação, avaliaram a produtividade e viabilidade econômica do cultivo e concluíram que a produtividade de melancia é fortemente influenciada pela disponibilidade de 
água.

ARAÚJO et al. (2007), em estudo de produtividade e viabilidade econômica do cultivo de melancia na região do submédio São Francisco, obtiveram produtividade média de $25.000 \mathrm{~kg} \mathrm{ha}^{-1}$, também inferiores aos obtidos em ConfresaMT. Essa elevada produtividade deve-se principalmente ao sistema de cultivo adotado, espaçamento, turno de rega, qualidade da água, e às rigorosas práticas de prevenção e controle de pragas e doenças, destacando-se o monitoramento intensivo de pragas e doenças, sendo que estas eram controladas prioritariamente no inicio da infestação. Além disso, foram realizadas aplicações dos fertilizantes de forma correta em época e quantidade.

Em Confresa-MT, a relação benefício/custo do cultivo foi de 1,86, ou seja, para cada $R \$ 1,00$ investido, tem-se retorno de $R \$ 1,86$ (Tabela 2). Essa baixa relação benefício/custo ocorre devido o elevado custo de produção na região de estudo. O custo foi calculado em $\mathrm{R} \$ 9.008,20 \mathrm{ha}^{-1}$, alavancado principalmente pelos custos com serviços que somaram $R \$ 4.310,00$ no custo de produção (Tabela 1).

SANDRI et al. (2014) verificaram que a relação benefício/custo do cultivo de melancia em Anápolis-GO variou de 1,9 em 2008 a 4,78 em 2010, quando era realizado o fornecimento de água via gotejamento. Assim, percebe-se que no ano de 2008, o valor foi de 1,9 e, apesar da relação ser baixa, ainda assim foi superior à obtida no presente estudo. Vale ressaltar que, no estudo realizado em Anápolis-GO, o custo com serviços foi inferior ao observado em Confresa-MT, causando menor impacto efetivo na relação.

TABELA 1: Custo de produção de um hectare de melancia irrigada em Confresa-MT, 2014. Descrição do custo Unidade Quantidade Valor Valor Total Unitário

\begin{tabular}{lcccc}
\hline INSUMOS & & & $\mathrm{R} \$$ & $\mathrm{R} \$$ \\
\hline Sementes & $\mathrm{kg} \mathrm{ha}^{-1}$ & 0,7 & 110,00 & 70,00 \\
Adubo orgânico (esterco) & $\mathrm{t} \mathrm{ha}^{-1}$ & 30 & 15,00 & 450,00 \\
Adubos & $\mathrm{kg} \mathrm{ha}^{-1}$ & 500 & 1,90 & 950,00 \\
Calcário & $\mathrm{kg} \mathrm{ha}^{-1}$ & 1600 & 0,25 & 400,00 \\
Adubo foliar & $\mathrm{L} \mathrm{ha}^{-1}$ & 5 & 22,50 & 112,50 \\
Fungicidas (benomyl) & $\mathrm{kg} \mathrm{ha}^{-1}$ & 2 & 80,00 & 160,00 \\
Inseticida & $\mathrm{L} \mathrm{ha}^{-1}$ & 2 & 60,00 & 120,00 \\
Óleo diesel & $\mathrm{L} \mathrm{ha}^{-1}$ & 50 & 2,9 & 145,00 \\
\hline Sub Total & & & & $\mathbf{2 . 4 0 7 , 5 0}$ \\
\hline SERVIÇOS & & & 120 & \\
\hline Aração e gradagem & $\mathrm{htt}$ & 4 & 120 & 120,00 \\
Conf. dos sulcos & $\mathrm{htt}$ & 1 & 60 & 120,00 \\
Conf. das covas & $\mathrm{dh}$ & 2 & 3,50 & 350,00 \\
Transporte & $\mathrm{km}$ & 90 & 60 & 360,00 \\
Adubação geral & $\mathrm{dh}$ & 6 & 60 & 900,00 \\
Capinas manuais & $\mathrm{dh}$ & 15 & 60 & 480,00 \\
Pulv. Manual & $\mathrm{dh}$ & 8 & 60 & 600,00 \\
Manut. da irrigação & $\mathrm{dh}$ & 10 & 60 & 900,00 \\
Colheita & $\mathrm{dh}$ & 15 & & $4.310,00$ \\
\hline
\end{tabular}

Sub total 4.310,00 


\begin{tabular}{|c|c|c|c|c|}
\hline Custo Operacional efetiv & & & & $6.717,50$ \\
\hline CUSTOS INDIRETOS & & & & \\
\hline Custo da terra & ha mês ${ }^{-1}$ & 3 & 650,00 & $1.950,00$ \\
\hline Administração & ha mês ${ }^{-1}$ & 3 & 55,00 & 165,00 \\
\hline Impostos e taxas & ha mês ${ }^{-1}$ & 3 & 38,23 & 114,69 \\
\hline Encargos Sociais & ha mês ${ }^{-1}$ & 3 & 11,84 & 35,52 \\
\hline $\begin{array}{l}\text { Depreciação do sistema } \\
\text { de Irrigação }\end{array}$ & ha mês ${ }^{-1}$ & 3 & 8,50 & 25,50 \\
\hline
\end{tabular}

Sub total

Custo total

$2.290,71$

dh corresponde a jornada diária de trabalho de um homem e htt corresponde a uma hora de trabalho de um trator.

A porcentagem dos custos com serviços atingiram $52,15 \%$ do custo operacional efetivo. Os elementos de despesa que mais impactaram no custo foram os procedimentos de controle das plantas daninhas por meio de capina, atividades de manutenção do sistema de irrigação e a colheita. Em estudo realizado em Goiatuba-GO, ASSUNÇÃO et al. (2014) também verificaram que os serviços corresponderam a $41,20 \%$ dos custos operacionais totais, envolvendo principalmente o manejo das plantas daninhas por meio da capina e o processo de colheita respondendo por $20,85 \%$ desse custo. Além disso, atividades como preparo de solo, gradagem e abertura de sulcos também representaram importante porcentagem dos gastos $(6,69 \%$ do total).

O ponto de nivelamento para o cultivo de melancia em Confresa-MT para o ano de 2014 foi de $25.737,74 \mathrm{~kg} \mathrm{ha}^{-1}$. Esse ponto de produtividade indica onde a receita se iguala aos custos, valor relativamente alto. Essa mesma interpretação pode ser verificada quando se avalia a margem de segurança, de -0,46. Esse índice determina que, para a receita se igualar à despesa, a quantidade produzida ou 0 preço do produto deverá cair até $46 \%$, na condição de estudo, ou seja, para o levantamento realizado no ano de 2014 , na região de Confresa-MT (Tabela 2).

De acordo com ARAÚJO et al. (2007), no vale do São Francisco em 2004, a margem de segurança foi de $-0,12$, situação em que no caso de uma variação negativa de $12 \%$ no preço do produto, o produtor já não obtém lucro no cultivo. Esse valor é quatro vezes menor que o verificado no presente estudo, demonstrando a diferença em função de sazonalidades, localização geográfica, fatores econômicos e outras variáveis, que interferem significativamente nesse índice.

Observa-se que mesmo com alta produtividade, próxima de $48.000 \mathrm{~kg} \mathrm{ha}^{-1}$, a receita líquida e relação benefício/custo são de certa maneira, elevadas, devido especialmente ao elevado custo de produção.

TABELA 2: Produtividade, receita bruta (RB), custo total da produção (CTP), receita 
liquida (RL), relação beneficio/custo $(R B / C)$, ponto de nivelamento (PN), margem de segurança (MS), do cultivo de melancia irrigada em Confresa-MT, 2014.

\begin{tabular}{|c|c|c|c|c|c|c|}
\hline Produtividade & RB & CTP & $\mathrm{RL}$ & $\mathrm{RB} / \mathrm{C}$ & PN & MS \\
\hline $\mathrm{kg} \mathrm{ha}^{-1}$ & $\mathrm{R} \$$ & $\mathrm{R} \$$ & $\mathrm{R} \$$ & & $\mathrm{~kg} \mathrm{ha}^{-1}$ & $\%$ \\
\hline $48.000,00$ & $16.800,0$ & $9.008,21$ & $7.791,79$ & 1,86 & $25.737,74$ & $-0,46$ \\
\hline $\begin{array}{l}\text { Produtividade mé } \\
\text { produzida (frutos } \\
\text { receita bruta/cust } \\
\text { da produção - rec } \\
\text { região de Confres }\end{array}$ & $\begin{array}{l}\text { produção } \\
\text { ruta/recei } \\
\text { em } 2014\end{array}$ & $\begin{array}{l}\text { I: custo da } \\
\text { ruta. Preçc } \\
\text { de } R \$ 0,3\end{array}$ & $\begin{array}{l}\text { ução/valor } \\
\text { io para a n } \\
\text { respectiva }\end{array}$ & $\begin{array}{l}\text { g cor } \\
\text { cia c } \\
\text { te. }\end{array}$ & $\begin{array}{l}\text { lizado; } M S \\
\text { ializada na }\end{array}$ & $\begin{array}{l}\text { mercial } \\
\text {; } \mathrm{RB} / \square \text { : } \\
\text { sto total } \\
\text { a para a }\end{array}$ \\
\hline
\end{tabular}

\section{CONCLUSÕES}

A cultivar Crimson select plus, na região de Confresa-MT, apresenta potencial de geração de renda, retorno financeiro relativamente bom e alta produtividade, sendo uma opção viável para os agricultores. de estudo.

O custo com serviços é o principal gargalo da produção de melancia na região

\section{REFERÊNCIAS}

ARAÚJO, J. L. P.; CORREIA, R. C.; SANTOS, R. F. Variação estacional do preço da melancia comercializada na região do Submédio São Francisco, período 1995-2005. Petrolina: Embrapa Semi-Árido, 2007. 4 p. (Embrapa Semi-Árido. Comunicado técnico, 133).

ASSUNÇÃO, P. E. V.; WANDER, A. E.; CARDOSO, J. S. Viabilidade econômica do sistema de produção de melancia no sul de Goiás. Conjuntura Econômica Goiana, v.29, n.1, p.32-46, 2014. Disponível em: $<$ http://www.imb.go.gov.br/down/conjuntura29.pdf>.

BHERING, M.C. et al. Avaliação do vigor de sementes de melancia (Citrullus lanatus Schrad) pelo teste de envelhecimento acelerado. Revista Brasileira de Sementes, v.25, n.2, p.1-6, 2003. Disponível em: $<$ http://www.scielo.br/scielo.php?script=sci_arttext\&pid=S010131222003000400001\&lng=pt\&nrm=iso\&tlng=pt>. doi: $10.1590 /$ S010131222003000400001.

CAMPELO, A.R.; AZEVEDO, B.M.; NASCIMENTO NETO, J.R.; VIANA, T.V.A.; PINHEIRO NETO, L.G.; LIMA, R.H. Manejo da cultura do melão submetida a frequências de irrigação e fertirrigação com nitrogênio. Horticultura Brasileira, v.32, n.2, p.138-144, 2014.

CEPEA. Melancia: clima prejudica melancicultura em 2015. Disponível em: http://www.cepea.org.br/hfbrasil/edicoes/152/melancia.pdf. Acesso em: 21 mai. 2016. 
DBCITY. Município de Confresa-MT. Disponível em: http://pt.db-city.com/Brasil-Mato-Grosso--Confresa. Acesso em: 15 mar. 2016.

DEEPASK. Agricultura: Veja produção agrícola e área plantada por cidade do Brasil - Confresa, MT. Disponível em: $<$ http://www.deepask.com/goes?page=confresa/MT-Agricultura:-Confira-a-producaoagricola-e-a-area-plantada-no-seu-municipio>. Acesso em: 07 abr. 2016.

EMBRAPA. Manejo e Conservação do Solo e da Água: Potencial Agrícola do Solo para 0 Cultivo da Melancia. Disponível em: <http://ainfo.cnptia.embrapa.br/digital/bitstream/CPATSA/35797/1/OPB1322.pdf>. Acesso em 08 jul. 2014.

FAO. Agricultural production: Primary crops. Rome, 2016. Disponível em:< http://www.fao.org>. Acesso em: 25 mai. 2016.

IBGE. Indicadores conjunturais; produção agrícola; agricultura. Disponível em: <http://biblioteca.ibge.gov.br/visualizacao/periodicos/66/pam_2014_v41_br.pdf>.

Acesso em: 21 mai. 2016.

MATSUNAGA, M.; BERNELMANS, P. F.; TOLEDO, P. E. N.; DULLEY, R. D.; OKAWA, H.; PEDROSO, I. A. Metodologia de custos de produção utilizada pelo IEA. Boletim Técnico do Instituto de Economia Agrícola, São Paulo, v.23, n.1, p. 123-139, 1976.

RIBEIRO, A. C.; GUIMARÃES, P. T. G.; ALVAREZ, V. H. V. Recomendações para o uso de corretivos e fertilizantes em Minas Gerais. CFSEMG, Viçosa-MG, 1999. $322 \mathrm{p}$.

SANDRI, D.; PEREIRA, J. A.; VARGAS, R. B. Custos de produção e rentabilidade produtiva da melancia sob diferentes lâminas e sistemas de irrigação. Irriga, v.19, n.3, p.414-429, $2014 . \quad$ Disponível em: <http://dx.doi.org/10.15809/irriga.2014v19n3p414>. doi: 10.15809/irriga.2014v19n3p414 